\title{
Prospective study of outcome in antenatally diagnosed renal pelvis dilatation
} Mervyn S Jaswon, Laraine Dibble, Sheila Puri, Jacky Davis, Jane Young, Raj Dave,
Heulwen Morgan viureteric junction (PUJ) obstruction, posterior urethral valves, and multicystic kidneys. Surgical uropathies feature highly, but this bias may be a reflection of the referral pattern of the tertiary paediatric urology units from which these studies come. ${ }^{34}$

Where there is an increase in the calibre of the renal pelvis at the site of the uretero-pelvic junction, but minimal or no dilatation of the intrarenal collecting system (renal pelvis dilatation) outcome, is less well documented. An association between antenatal renal pelvis dilatation (ARPD) and vesicoureteric reflux (VUR) ${ }^{5}$ is particularly important in view of the association between VUR and the subsequent development of renal scars.

However, the incidence of this association and the correct postnatal investigation of these cases has not been fully established. Furthermore, there is no consensus on the measurements for a normal fetal renal pelvis. Consequently, the threshold for investigation differs among hospitals.

We therefore conducted a prospective investigation in a district general hospital (with no bias in its referral pattern). All babies were enrolled whose routine antenatal scan showed that the anterioposterior diameter of one or both renal pelves was $5 \mathrm{~mm}$ or above. The babies were investigated postnatally to determine whether there was any significant abnormality associated with the ARPD and to ascertain whether an upper limit of normal for the fetal renal pelvis could be defined.

Keywords: vesicoureteric reflux; antenatal renal pelvi dilatation; renal tract anomalies; prenatal diagnosis

Anomalies are reported in around $2-3 \%$ of routine antenatal ultrasound scans ${ }^{1}$; about a third of these are due to abnormalities of the renal tract. Hydronephrosis detected antenatally was first reported in the early 1980s, since when there have been many case reports and studies describing the postnatal diagnosis associated with these findings. ${ }^{2}$ In these studies there has been a preponderance of cases of pel-

\section{Methods}

The study was conducted over 26 months, during which time there were 7000 deliveries. All infants whose 20 week anomaly scan showed that one or both renal pelves was $5 \mathrm{~mm}$ or greater (anterioposterior diameter) were entered. Patients $(<10 \%)$ who had only renal pelvis dilatation (RPD) on subsequent scans, which had been done for obstetric reasons,
Department of

Radiology

J Davis

J Young

R Dave

Department of Obstetrics and

Gynaecology

H Morgan

Correspondence to: Dr Jaswon.

Accepted 11 September 1998

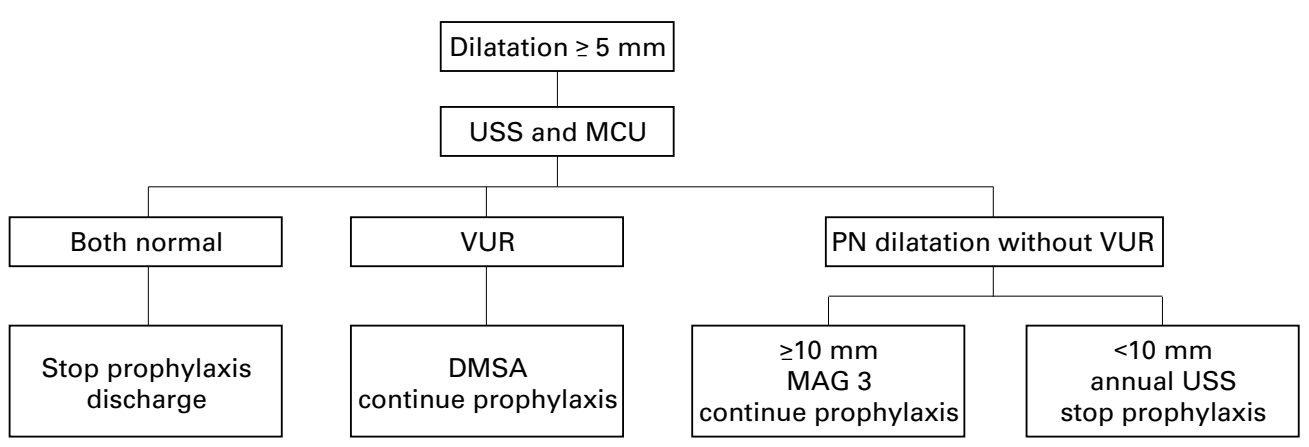

Figure 1 Protocol for investigation of children with antenatal renal pelvis dilatation. 
were also enrolled in the study. Investigation followed the protocol set out in fig 1 .

At the antenatal scan parents of eligible infants were given an explanatory letter with a diagram outlining the possible clinical significance of the ultrasound findings, as well as the rationale for postnatal investigation. They were also advised that they could talk to one of the paediatricians involved (MSJ, LD) for further clarification, if they so wished.

A urine sample was obtained at birth and the baby started on antibiotic prophylaxis. An ultrasound scan was performed between two and six weeks after delivery, except for two infants who had scans when they were 3 and 4 days old. A micturating cystogram (MCU) was performed at two to three months (in girls not before 3 months for technical ease). All babies had an MCU whatever the scan results. The baby was then reviewed in clinic, growth and blood pressure measured, and a further urine sample obtained.

If both the postnatal scan and MCU were normal and the general review was satisfactory, antibiotic prophylaxis was discontinued and the infant discharged from further follow up. If the MCU showed the presence of VUR, antibiotic prophylaxis was continued and a ${ }^{99} \mathrm{~m}$ technetium labelled dimercaptosuccinic acid scan (DMSA) was performed.

VUR was graded as follows: (I) reflux into the distal ureter, often transient; (II) reflux into the ureter, renal pelvis, and calyces with no dilatation; (III) reflux with moderate dilatation of the ureter, renal pelvis, and calyces; (IV) reflux with gross dilatation and tortuosity of the ureter, gross dilatation of the renal pelvis, and calyceal clubbing.

When the MCU was normal but the postnatal scan showed persistent RPD, further management depended on the degree of dilatation. Where the RPD was $\geqslant 10 \mathrm{~mm}$ a ${ }^{99 \mathrm{~m}}$ technetium labelled mercapto-acetyl triglycine renogram (MAG 3) with diuretics (frusemide $0.5 \mathrm{mg} / \mathrm{kg}$ ) was performed to assess renal drainage and function. Where RPD measured 5-10 $\mathrm{mm}$ a further ultrasound scan was arranged when the infant was a year old.

The antenatal scans were performed by a senior radiographer (RD) using either an ATL UM4 scanner with a $4.0 \mathrm{MHz}$ linear transducer, or an ALOKA SSD-650 with a curvilinear, multifocusing $3.5 \mathrm{MHz}$ transducer. The postnatal scans were performed by a consultant radiologist using an ATL HDI 3000 machine, with a $4-7 \mathrm{MHz}$ broadband, multifrequency, multifocusing transducer.

Ethical approval for the study was granted by the local ethical committee.

\section{Results}

One hundred and thirty nine mothers were enrolled prospectively during the study period (May 1994 to July 1996). Their babies were booked for investigation following the above protocol and investigations were completed in 104 babies. Twenty one babies failed to attend or had incomplete investigations and seven moved out of the area during the study and were lost to follow up. In five cases the parents

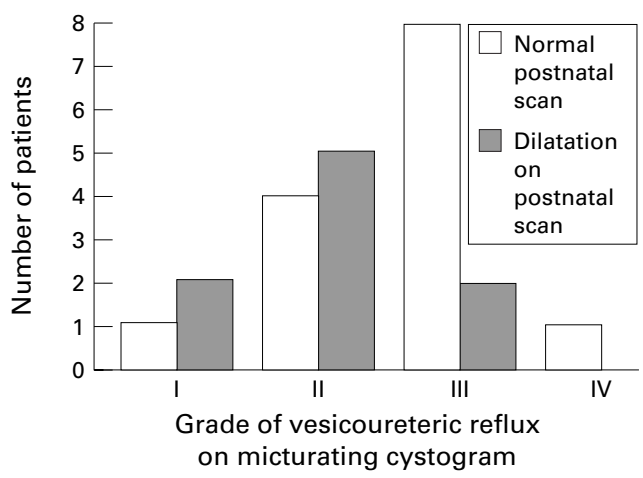

Figure 2 Correlation between grade of vesicoureteric reflux and findings at postnatal ultrasound scan.

Table 1 Diagnosis in infants with postnatal renal pelvis dilatation

\begin{tabular}{|c|c|c|}
\hline Diagnosis & $N=$ & Comments \\
\hline VUR & 9 & No scarring in refluxing kidney \\
\hline $\begin{array}{l}\text { Idiopathic } \\
\text { dilatation }\end{array}$ & 8 & $\begin{array}{l}\text { No VUR or PUJ obstruction, RPD } \\
10-20 \mathrm{~mm}\end{array}$ \\
\hline PUJ obstruction & 4 & $\begin{array}{l}\text { (i) One kidney showed loss of } \\
\text { function and underwent pyeloplasty } \\
\text { (ii) Three had no loss of function and } \\
\text { were managed conservatively }\end{array}$ \\
\hline Renal dyplasia & 4 & $\begin{array}{l}\text { Contralateral kidney: } \\
\text { (i) VUR } \\
\text { (ii) Mild hydronephrosis -? crossed } \\
\text { ectopia } \\
\text { (iii) Hypertrophic }\end{array}$ \\
\hline
\end{tabular}

VUR=vesicoureteric reflux; PUJ=pelviureteric junction; $\mathrm{RPD}=$ renal pelvis dilatation.

elected not to follow our protocol. Two babies were excluded from the study (termination because of trisomy 21, stillbirth).

VUR was the most common clinical significant pathology $(23 / 104$ or $22 \%)$. There was no correlation between the degree of either anteor postnatal renal pelvis dilatation (RPD) and the severity of VUR. The postnatal ultrasound scan was normal in 14/23 (61\%) of infants who had VUR. Fig 2 shows that the degree of VUR was, in fact, worse in children with a normal postnatal ultrasound scan than in those with dilatation on the postnatal scan. In one infant with grade IV the anomaly scan was normal but in subsequent scans (at 32 and 36 weeks gestation) performed for obstetric reasons RPD was noted and VUR diagnosed postnatally.

The DMSA scan showed no evidence of scarring or renal dysplasia in any of the refluxing units. Reduced renal function $(34 \%)$ was found in the contralateral side to one refluxing kidney. There was a male and right sided preponderance in infants diagnosed with VUR.

In $47(45 \%)$ infants persisting renal pelvis dilatation was identified on the postnatal scan, and the outcome in these cases is detailed in table 1 .

Idiopathic dilatation of $\geqslant 10 \mathrm{~mm}$-persisting dilatation in the presence of a normal MCU and MAG 3-was found in eight cases (7\%). There were four cases of PUJ obstruction, defined by delay in excretion (less than $50 \%$ in 10 minutes) on MAG 3, none of whom had significant renal impairment, initially, and were managed conservatively. However, one of the cases went on to have a pyeloplasty at 18 months because of deteriorating renal function. 
Four infants had renal dysplasia, three of whom also had an abnormality affecting the contralateral kidney (VUR, mild hydronephrosis, hypertrophic kidney). This highlights the need to investigate the contralateral kidney in infants with renal dysplasia (table 1).

There was generally a high uptake of postnatal investigations, notably in those parents who had attended for antenatal counselling. Sixty eight of $139(49 \%)$ parents attended for counselling, and of this group seven (10\%) infants failed to attend for postnatal investigation, in contrast to $14(20 \%)$ infants from those parents who were not counselled.

\section{Discussion}

Renal tract abnormalities detected on antenatal ultrasound scan are relatively common and occurred in $2 \%$ of pregnancies in our prospective and unselected series. There are few data on outcome, or appropriate management, of minor degrees of antenatal renal pelvis dilatation, which comprise most cases in a district general hospital. In our population VUR was the most common postnatal diagnosis, occurring in $22 \%$ of cases. We found four cases (3.8\%) of PUJ obstruction, generally the most common diagnosis in reports of antenatal hydronephrosis.

Our study confirms the findings of Dudley et $a l^{6}$ that vesicoureteric reflux is the most common finding in the milder spectrum of ARPD. However, our investigation protocol differed significantly in that we performed micturating cystograms on all babies even if the postnatal ultrasound scan was normal. Thus a diagnosis of VUR was made in 14 babies who had a normal postnatal ultrasound scan. The importance of looking for reflux, even in the presence of a normal postnatal ultrasound scan, is supported by the findings of Zerin et $a l,{ }^{7}$ and Tibballs. ${ }^{8}$ However, both of these were retrospective studies looking at a selected population referred to a specialist centre.

In six children subsequently shown to have VUR only one out of several antenatal scans was abnormal. Thus a single antenatal measurement which shows evidence of renal pelvis dilatation seems to put the fetus into the risk category for VUR.

The importance of parental information, explanation, and counselling, as highlighted by Watson $e t a l^{9}$ and Owen, ${ }^{10}$ is supported in our study. Out of 68 couples $(49 \%)$ who came to discuss the antenatal findings, only seven $(10 \%)$ failed to complete postnatal investigation; of those who did not have any antenatal counselling, the baby was not investigated in 14 $(20 \%)$ cases, though it must be acknowledged that there is an element of self selection in those parents attending for counselling. There is a danger of engendering unnecessary anxiety by covering in detail all possible outcomes, particularly where there are likely to be a significant number of false positive results. ${ }^{11}$ However, this is not an argument against counselling, nor should it act as deterrent. Similar dilemmas are encountered in many branches of medicine, but particularly in the field of antenatal diagnosis where ultrasonography identifies "soft markers" of fetal anomaly. ${ }^{12}$

The male preponderance in this series, and in other studies, contrasts with the female bias in postnatally detected VUR and might support the hypothesis that the VUR detected on the basis of ARPD, as in our study, is a different entity. However, as discussed by Marra, ${ }^{13}$ this difference in sexual bias may be a reflection of case presentation, rather than incidence, because in postnatally detected VUR the event leading to its diagnosis is a urinary tract infection, which is more common in girls. Further information regarding kindred clustering of VUR detected on the basis of ARPD would be helpful in resolving this issue, because in cases of postnatally diagnosed VUR there is a definite familial bias, with around a $30 \%$ incidence in siblings.

Anderson and Rickwood ${ }^{14}$ have suggested that VUR detected on the basis of ARPD may be a marker of a more generalised poor renal tract development. They found renal impairment, detected by DMSA, in $75 \%$ of cases of ARPD and VUR, in the absence of documented infection, and commented that this represented dysplastic kidneys due to a prenatal vascular accident affecting renal tract development. However, in our study none of the refluxing units showed any evidence of renal impairment.

The rationale for intervention in this area assumes that VUR detected on the basis of ARPD is the same condition as that found in children with urinary tract infections, and therefore has the same clinical significance in terms of the pathogenesis of renal scarring and its long term sequelae. It could be argued that the VUR detected in our study is a phenomenon of fetal renal tract development (transient renal tract dilatation) and is a self limiting condition which could well be left alone, or is merely detecting the background incidence of VUR. However, the prevalence of asymptomatic (and presumably clinically insignificant) VUR as reviewed by Bailey ${ }^{15}$ is $0.8-1.4 \%$, compared with our figures of over $20 \%$. We therefore consider that VUR detected on the basis of ARPD represents the detection of a pathological condition which may lead to renal scarring and justifies our investigative protocol.

We also set out to determine an upper limit of normal for the antenatal renal pelvis. However, as VUR was shown in association with all degrees of ARPD from $5 \mathrm{~mm}$ and upwards, it has not been possible to determine an upper limit of normal for the antenatal renal pelvis. In conclusion, we suggest that a measurement of $5 \mathrm{~mm}$, or above, of the fetal renal pelvis is an indication for postnatal investigation, including MCU, notwithstanding the presence of a normal postnatal ultrasound scan.

1 Chitty LS, Hunt GH, Moore J, Lobb MO. Effectiveness of routine ultrasonography in detecting fetal structural abnormalities in a low risk population. BMF 1991;303:1165-9.

2 Harrison MR, Golbus MS, Filly RA, et al. Management of the fetus with congenital hydronephrosis. $\mathcal{F}$ Paed Surg 1982;17:728-40. 
3 Dell'Agnola CA, Tomaselli V, Ferazzi J, et al. Perinatal ultrasound monitoring: early detection and treatment of congenital uropathy. Brf Urol 1983;55:469-72.

4 Thomas DFM, Gordon AC. Management of prenatally diagnosed uropathies. Arch Dis Child 1989;64:58-63.

5 Najmaldin A, Burge DM, Atwell JD. Fetal vesicoureteric reflux. Br f Urol 1990;65:403-6.

6 Dudley JA, Haworth JM, McGraw ME, Frank JD, Tizard EJ. Clinical relevance and implications of antenatal hydronephrosis. Arch Dis Child 1997;76:F31-4

7 Zerin JM, Ritchey ML, Chang ACH. Incidental vesicouretric reflux in neonates with antenatally detected hydronephrosis and other renal abnormalities. Radiology 1993; 187:157-60.

8 Tibballs JM, De Bruyn R. Primary vesicoureteric reflux how useful is postnatal ultrasound? Arch Dis Child 1996; 75:444-7.

9 Watson AR, Readett D, Nelson CS, Kapila L, Mayell MJ. Dilemmas associated with antenatally detected urinary tract abnormalities. Arch Dis Child 1988;63:719-22.
10 Owen RJT, Lamont AC, Brookes J. Early management and postnatal investigation of prenatally diagnosed hydronephrosis. Clin Radiol 1996;51:173-6.

11 Marteau TM, Cook R, Kidd J, et al. The psychological effects of false-positive results in prenatal screening for fetal abnormality:a prospective study. Prenatal Diagn 1992;12:205-14.

12 Whittle M. Ultrasonographic "soft markers" of fetal chromosomal defects. BMF 1997;314:918.

13 Marra G, Barbieri G, Moioli C, Assael BM, Grumieri G, Caccamo ML. Mild fetal hydronephrosis indicating vesicoureteric reflux. Arch Dis Child 1994;70:F147-F50.

14 Anderson PAM, Rickwood AMK. Features of primary vesicoureteric reflux detected by prenatal sonography. $\mathrm{Br} F$ Urol 1991;67:267-71.

15 Bailey RR. Vesicoureteric reflux in healthy infants and children. In:Hodson J, Kincaid Smith P, eds. Reflux Nephropathy. New York: Masson, 1979:57-61. 